\title{
Effect of formulation and repeated applications on the enantioselectivity of metalaxyl dissipation and leaching in soil
}

Rafael Celis,* Beatriz Gámiz, María A Adelino, Juan Cornejo and María C Hermosín

Instituto de Recursos Naturales y Agrobiología de Sevilla (IRNAS), CSIC, Avenida Reina Mercedes 10, P.O. Box 1052, 41080 Sevilla, Spain

Running Title: Enantioselective behavior of metalaxyl in soil under repeated applications

*Corresponding Author: Dr. Rafael Celis

Address: Instituto de Recursos Naturales y Agrobiología de Sevilla (IRNAS), CSIC Avenida Reina Mercedes 10, P.O. Box 1052

41080 Sevilla, Spain

Phone: +34 954624711

Fax: $\quad+34954624002$

E-mail: rcelis@irnase.csic.es 


\section{Abstract}

2 BACKGROUND: Soil incubation and column leaching experiments were conducted to address the question of whether the type of formulation (unsupported vs clay-supported) and repeated applications of the chiral fungicide $(R S)$-metalaxyl affected the enantioselectivity of its dissipation and leaching in a slightly alkaline, loamy sand agricultural soil. RESULTS: Regardless of the type of formulation and the number of fungicide applications, the $R$-enantiomer of metalaxyl was degraded faster than the $S$-enantiomer, but the individual degradation rates of $R$ - and $S$-metalaxyl were highly affected by the different application regimes assayed $\left(t_{1 / 2}=2-104\right.$ days). Repeated applications accelerated the degradation of the mixtures of enantiomers or racemates to agricultural soils. hydroxides 


\section{$1 \quad 1$ INTRODUCTION}

2 Improving the current knowledge on the enantioselectivity of processes determining the fate of chiral pesticide enantiomers in the soil environment is necessary to maximize the efficacy and minimize the environmental impact of chiral pesticides, as well as to address the suitability of replacing pesticide racemic mixtures with single biologically-active enantiomers for a more rational pesticide use. ${ }^{1-4}$ Nevertheless, chiral pesticide enantiomers are often treated as a single compound in standard chemical analyses, and for economic reasons, they are primarily used as racemic mixtures or racemates, because large scale production of single pesticide enantiomers is usually much more expensive than the production of the corresponding pesticide racemic mixture. ${ }^{5,6}$ The fact that most chiral pesticides are used, regulated, and analyzed as if they were achiral also reflects the lack of knowledge about their enantioselective environmental behavior, since most studies on chiral pesticides do not explicitly account for individual stereoisomers, and consequently, they implicitly and incorrectly assume that enantiomers have identical environmental behavior. ${ }^{6,7}$ Addressing the enantioselective environmental behavior of chiral pesticides has only recently become a growing area of research, particularly when chiral liquid chromatography columns providing good resolving power to separate enantiomers have become more readily commercially available than in the past. ${ }^{3,4}$

It is well known that, despite the chemical similarity of the enantiomeric forms of a chiral pesticide, the biological activities of chiral pesticide enantiomers are usually very different. The herbicidal activity of the $R(+)$-enantiomer of imidazolinone herbicides is about eight times greater than that of the $S(-)$-enantiomer, and for the herbicide metolachlor, which has two chiral centers, the two $S$-enantiomers are about ten times more toxic to target weeds than the two $R$-enantiomers. ${ }^{2,8}$ This is due to the fact that the final molecular target of a pesticide is in most cases a biological receptor, e.g. an enzyme, whose active center is also chiral and in 
1 turn enantioselective. ${ }^{1,2,6,8}$ Similarly, the degradation of chiral pesticides in soil is often enantioselective, primarily due to the enantioselectivity of the soil biological degradation processes. ${ }^{9-13}$ For example, the herbicide dichlorprop was shown to be enantioselectively transformed in the surface soil after application to an experimental field, with the (-)enantiomer exhibiting a half-life of $\sim 4$ days and the (+)-enantiomer $\sim 8$ days. ${ }^{10}$ Abiotic processes, such as chemical degradation and soil sorption/transport processes, are often assumed to be non-enantioselective, ${ }^{9}$ although several authors have presented evidence that different chiral organic compounds can exhibit strong optical isomer selectivity in their sorption on natural soil and sediment materials. ${ }^{14-16}$ Furthermore, despite being intrinsically non-enantioselective processes, leaching and runoff of chiral pesticides could show an apparent enantiomer-selectivity if one of the enantiomers becomes more available for transport than the other as a result of being less sorbed or degraded. ${ }^{13,17}$ Metalaxyl [methyl- $N$-(2-methoxyacetyl)- $N$-(2,6-xylyl)- $D L$-alaninate] is a chiral acylanilide fungicide widely used in the control of plant diseases caused by pathogens of the Oomycota division in several crops. Its fungicidal activity mostly originates from the $R(-)$-enantiomer. ${ }^{6,12}$ Previous studies have reported that metalaxyl sorption by soils and soil components appears to be mainly non-enantioselective, whereas soil degradation of metalaxyl is an enantioselective, microbiologically-mediated process, with $S$-metalaxyl being degraded more rapidly than $R$-metalaxyl in anaerobic soils and aerobic soils with $\mathrm{pH}<4$, and $R$-metalaxyl being degraded more rapidly than $S$-metalaxyl in aerobic soils with $\mathrm{pH}>5 .{ }^{12,13,18-20}$ Sorption and entrapment in small-size pores were suggested to reduce the availability of metalaxyl enantiomers to microbial degradation and hence the enantioselective degradation of the fungicide $;^{13}$ the less available metalaxyl residues appeared to remain racemic longer than the fraction readily available to soil microorganisms. ${ }^{13}$ 
The form in which a pesticide is applied to soil and repeated pesticide applications to the same soil are agricultural practices that can greatly influence pesticide dissipation and leaching patterns. For example, accelerated degradation after repeated pesticide applications and reduced leaching by the use of clay-based formulations, compared to formulations containing the pesticide in an immediately available form, have been described. ${ }^{21-30}$ For chiral pesticides, research conducted on this subject does not generally account for individual enantiomers, implicitly assuming that the effects of such practices are identical for both enantiomers. $^{31,32}$

The main objective of this work was to address the question of whether repeated applications could affect the enantioselectivity of metalaxyl dissipation and leaching in soil. Water-dissolved metalaxyl and two novel clay-based formulations of the fungicide were used in the experiments to further address the effect of formulation on the enantioselectivity of metalaxyl dissipation and leaching patterns under single and repeated fungicide application regimes. The clay-based formulations were prepared by pre-adsorbing the fungicide on hydrotalcite (anionic clay) treated with oleate and elaidate anions (HT-OLE and HT-ELA). Several studies have shown that modification of hydrotalcite-like compounds with organic anions can increase the hydrophobicity of these minerals, yielding nanostructured hybrid materials (organohydrotalcites) with high affinities for different types of organic compounds. ${ }^{33-37}$ As a result of this behavior, organohydrotalcites were proposed as supports for the design of pesticide slow release formulations with the purpose of reducing pesticide leaching in soil. ${ }^{30}$

\section{MATERIALS AND METHODS}

\subsection{Fungicide and soil}

High-purity, analytical standard grade, racemic metalaxyl (chemical purity > 99.5\%) purchased from Sigma-Aldrich (Spain) was used in the experiments. The soil used was a 
1 slightly alkaline, agricultural soil (loamy sand) dedicated to winter cereal from an

experimental farm located in Seville (SW Spain, $37^{\circ} 17^{\prime} \mathrm{N}, 6^{\circ} 3^{\prime} \mathrm{W}$ ). It had $83 \%$ sand, $7 \%$ silt, $10 \%$ clay (3\% smectites, $5 \%$ illite/mica, $2 \%$ kaolinite), $0.54 \%$ organic $\mathrm{C}, 6.5 \% \mathrm{CaCO}_{3}$ and $\mathrm{pH}$ of 7.5 . The soil was sampled from the $0-20 \mathrm{~cm}$ soil layer, air-dried, and sieved to pass a $2 \mathrm{~mm}$ mesh before used.

\subsection{Preparation of the sorbents and assessment of their affinity for metalaxyl}

Two clay-based formulations of metalaxyl were prepared for this study by pre-adsorbing the fungicide on 3:1 Mg/Al layered double hydroxide, also known as hydrotalcite (HT) or anionic clay, ${ }^{38}$ intercalated with oleate and elaidate anions, respectively.

Hydrotalcite (HT) was prepared by adding dropwise an aqueous solution (100 mL) containing $\mathrm{Mg}\left(\mathrm{NO}_{3}\right)_{2} \cdot 6 \mathrm{H}_{2} \mathrm{O}(0.3 \mathrm{~mol})$ and $\mathrm{Al}\left(\mathrm{NO}_{3}\right)_{3} \cdot 9 \mathrm{H}_{2} \mathrm{O}(0.1 \mathrm{~mol})$ to an alkaline solution $(500 \mathrm{~mL})$ containing $\mathrm{NaOH}(1.6 \mathrm{~mol})$ and $\mathrm{Na}_{2} \mathrm{CO}_{3}(0.37 \mathrm{~mol})$. The resulting precipitate was hydrothermally treated $\left(80^{\circ} \mathrm{C}, 24 \mathrm{~h}\right)$, washed with deionized water, and then freeze-dried.

Oleate- and elaidate-intercalated organohydrotalcite samples (HT-OLE and HT-ELA) were prepared by the reconstruction method using calcined-HT or HT500, i.e. the product resulting from heating the HT sample at $500{ }^{\circ} \mathrm{C}$ for $3 \mathrm{~h}$, following the procedure described in Celis et al. ${ }^{33}$ For the synthesis, HT500 (500 mg) was added to an aqueous solution (100 mL) containing 3 mmol of oleic acid or elaidic acid (Sigma-Aldrich, Spain) and $\mathrm{NaOH}$ (3.3 mmol). The suspensions were shaken for $24 \mathrm{~h}$ at $60^{\circ} \mathrm{C}$, and the resulting organohydrotalcites (HT-OLE and HT-ELA) were washed with deionized water, and then air-dried. A detailed description of the structural characteristics and sorptive properties of HT-OLE and HT-ELA can be found in Celis et al. ${ }^{33}$

Metalaxyl sorption isotherms on the unmodified (HT) and modified (HT-OLE and HTELA) clay samples were obtained by equilibrating ( 24 h-shaking at $20 \pm 2{ }^{\circ} \mathrm{C}$ ) triplicate 
1 sorbent samples $(20 \mathrm{mg})$ with metalaxyl solutions $(8 \mathrm{~mL})$ with initial fungicide concentrations

ranging between 0.1 and $600 \mathrm{mg} / \mathrm{L}$. Once equilibrated, the suspensions were centrifuged, filtered, and the supernatants were analyzed by chiral HPLC (see below) to determine the equilibrium concentrations of $S$ - and $R$-metalaxyl. The amounts sorbed were calculated from the difference between the initial and equilibrium solution enantiomer concentrations.

\subsection{Preparation of the clay-based formulations of metalaxyl and determination of the}

\section{fungicide release patterns}

The two organohydrotalcite-based formulations of metalaxyl were prepared by pre-adsorbing the fungicide on HT-OLE and HT-ELA, respectively. For this purpose, HT-OLE or HT-ELA (200 mg) was added to a $600 \mathrm{mg} / \mathrm{L}$ aqueous metalaxyl solution $(80 \mathrm{~mL})$. The suspensions were shaken $(24 \mathrm{~h})$ at $20 \pm 2{ }^{\circ} \mathrm{C}$, centrifuged, filtered $(0.45 \mu \mathrm{m})$, and the resulting organohydrotalcite-metalaxyl formulations were air-dried, homogenized in an agate mortar, and stored at room temperature until used. The amount of metalaxyl in the formulations was calculated from the difference between the amount of fungicide added and that remaining in solution after shaken with the sorbents, and was in agreement with the amount directly determined by extracting the formulations $(5 \mathrm{mg})$ with methanol $(10 \mathrm{~mL})$. These fungicide contents in the formulations were $4.4 \%$ for HT-OLE and $5.6 \%$ for HT-ELA.

The kinetics of metalaxyl release into water from its complexes with HT-OLE and HTELA was determined in triplicate by suspending $0.5 \mathrm{mg}$ of metalaxyl (active ingredient, a.i.), as HT-OLE-Metalaxyl complex $(11.4 \mathrm{mg}$ ) or HT-ELA-Metalaxyl complex $(8.9 \mathrm{mg})$, in water $(250 \mathrm{~mL})$ using glass bottles closed with screw caps. ${ }^{30}$ At selected times, the bottles were hand-shaken, the contents were allowed to settle $(10 \mathrm{~min})$, and an aliquot of the supernatant solution ( $3 \mathrm{~mL}$ ) was sampled, filtered, and analyzed by chiral HPLC to determine the concentration of $S$ - and $R$-metalaxyl. 


\subsection{Dissipation experiment}

We studied the enantioselective aerobic dissipation of metalaxyl in the test soil upon three successive applications of the fungicide either as free (water-dissolved) pure compound or as organohydrotalcite-based formulations. Soil samples (200 g) were spiked with rac-metalaxyl (free, HT-OLE, or HT-ELA formulation) at a rate of $2 \mathrm{mg}$ a.i./kg soil, and then incubated in glass jars at $20 \pm 2{ }^{\circ} \mathrm{C}$ for 42 days. The soil moisture content was maintained at a constant level $(25 \%)$ by adding deionized water as necessary. Periodically $(0,1,7,14,21,28,35$ and 42 days after treatment, DAT), triplicate soil aliquots ( $3 \mathrm{~g})$ were sampled using a sterilized spatula and immediately frozen until analyzed. The amounts of $S$ - and $R$-metalaxyl in the $3 \mathrm{~g}$ soil aliquots were determined by extraction with methanol $(8 \mathrm{~mL})$, followed by centrifugation, and enantioselective analysis of the supernatant solution by chiral HPLC. Preliminary experiments had shown that this extraction procedure recovered $>95 \%$ of the $S$ - and $R$ metalaxyl freshly applied to the soil, so that the amounts not recovered during the incubation study were assumed to be degraded. It is important to note that metalaxyl has been shown to be configurationally stable in soil, showing no interconversion of $R$ - to $S$-enantiomers, and vice-versa. $^{12,39}$

After completing the 42-day incubation period corresponding to the first application experiment, the free (water dissolved) or HT-OLE/HT-ELA formulations of rac-metalaxyl were again applied to the soil at a rate of $2 \mathrm{mg}$ a.i./ $\mathrm{kg}$. The spiked soil was incubated for an additional period of 26 days and it was periodically sampled $(0,1,5,12,19$ and 26 DAT) and analyzed as described for the first application experiment. Finally, a third application of racmetalaxyl to the soil was conducted after completing the 26 day incubation period of the second application experiment. The soil was incubated again for 42 days, and periodically sampled $(0,1,4,7,11,14,18,21,28,35$ and 42 DAT) and analyzed. 
At each sampling time, the enantiomer fraction $(E F)$ was calculated by dividing the soil concentration of $S(+)$-metalaxyl by the sum of the soil concentrations of the $S(+)$ - and $R(-)$ metalaxyl enantiomers. ${ }^{40}$ In addition, $S$ - and $R$-metalaxyl dissipation data were fitted to the linearized form of the single first-order kinetic equation:

$$
\ln C=\ln C_{0}-k t
$$

where $C(\mathrm{mg} / \mathrm{kg})$ and $C_{0}(\mathrm{mg} / \mathrm{kg})$ are the enantiomer concentration in soil at time t (days) and time zero from the corresponding fungicide application, respectively, and $k\left(\right.$ days $\left.^{-1}\right)$ is the single first-order dissipation rate constant. SigmaPlot 12.5 software was used to calculate the slopes $(k)$ and intercepts $\left(\ln C_{0}\right)$ of the linear regressions of $\ln C$ versus $t$, and also to plot the experimental and fitted dissipation curves. An analysis of covariance (ANCOVA) was then performed (IBM SPSS Statistics 22) to compare pair-wise the slopes of the regression lines (k). The half-lives $\left(t_{1 / 2}\right)$ of $S$ - and $R$-metalaxyl were calculated as $t_{1 / 2}=0.693 / k$.

\subsection{Column leaching experiment}

Leaching experiments were conducted in glass columns of $30 \mathrm{~cm}$ length $\times 3.1 \mathrm{~cm}$ internal diameter filled with air-dried soil. The top $5 \mathrm{~cm}$ of the columns were filled with sea sand (10 $\mathrm{g})$ and the bottom $5 \mathrm{~cm}$ with sea sand $(10 \mathrm{~g})$ plus glass wool, to prevent losses of soil during the experiment. The rest of the column $(20 \mathrm{~cm})$ was hand-packed with soil $(160 \mathrm{~g})$. The soil columns were saturated with water and allowed to drain for $24 \mathrm{~h}$. The amount of water retained by the soil columns after saturation (column pore volume, $\mathrm{V}_{\mathrm{p}}$ ) was $57 \pm 2 \mathrm{~mL}$. Rac-metalaxyl was applied to the top of the soil columns at a rate of $2 \mathrm{~kg} / \mathrm{ha}$ as $0.15 \mathrm{mg}$ of active ingredient either dissolved in $1 \mathrm{~mL}$ of water or pre-adsorbed on HT-OLE and HT-ELA. After applying the fungicide, $15 \mathrm{~mL}$ of deionized water was applied daily to the top of the soil 
1 columns and the leachates were collected, filtered, and analyzed by chiral HPLC to determine the concentration of $S$ - and $R$-metalaxyl. After a total of 17 additions of $15 \mathrm{~mL}$ of water $(255$ $\mathrm{mL}$ of water in 17 days), a second application of metalaxyl to the soil columns was performed, either as water-dissolved fungicide or as HT-OLE/HT-ELA formulations. Daily additions of $15 \mathrm{~mL}$ of water to the top of the soil columns were again carried out for a total of 17 water additions. The leachates were collected, filtered, and analyzed by chiral HPLC, as described for the first application experiment. Finally, a third application of metalaxyl was conducted after completing the 17 water additions of the second application experiment, following the same experimental procedure as that described for the previous applications. All treatments were conducted in triplicate.

\subsection{Analysis of metalaxyl enantiomers}

Metalaxyl enantiomers were determined by chiral HPLC using a Waters 600E chromatograph coupled to a 996 diode-array detector. The chromatographic conditions used for the analysis are described in detail in Celis et al. ${ }^{13}$ Briefly, we used a Chiralpak IB column (150 mm length $\times 4.6 \mathrm{~mm}$ i.d., $5 \mu \mathrm{m}$ particle size), 60:40 (v.v) water:acetonitrile eluent mixture at a flow rate of $1 \mathrm{~mL} / \mathrm{min}, 50 \mu \mathrm{L}$ sample injection volume, and UV detection at $213 \mathrm{~nm}$. External calibration curves with five standard solutions between 0.1 and $6 \mathrm{mg} / \mathrm{L}$ of $\mathrm{rac}$-metalaxyl were used in the calculations. Under these conditions, $S(+)$ - and $R(-)$-metalaxyl eluted at 4.9 and $6.1 \mathrm{~min}$, respectively, and rac-metalaxyl was found to yield an exact peak area ratio of 1:1 $(E F=0.500 \pm 0.003)$ in both standard solutions and freshly spiked aqueous and methanolic soil extracts. ${ }^{13}$ The limit of quantification (LOQ) was $0.02 \mathrm{mg} / \mathrm{L}$ for both enantiomers.

\section{RESULTS AND DISCUSSION}

\subsection{Metalaxyl formulations}


1 Data in Figure 1 confirm the great enhancement in metalaxyl sorption by hydrotalcite upon its

2 modification with elaidate and oleate anions (Figure 1a), as well as the sustained release of

3 the fungicide from the formulations prepared in this work when suspended in pure water

4 (Figure 1b). The behavior of both formulations was similar, although HT-ELA displayed

5 slightly greater sorption and slower release of the fungicide compared to HT-OLE. As

6 expected from the non-enantioselective nature of the sorbents, both sorption and release

7 processes were found to be non-enantioselective, with the $S$-metalaxyl enantiomer being sorbed and released to the same extent as the $R$-enantiomer. Metalaxyl concentration data given in Figure 1a and $1 \mathrm{~b}$ represent the sum of $S+R$ metalaxyl sorbed and released, respectively.

\subsection{Metalaxyl dissipation}

Figure 2 shows the successive dissipation curves for $S$ - and $R$-metalaxyl in the soil along the incubation experiment, in which rac-metalaxyl was repeatedly applied (three times) at a rate of $2 \mathrm{mg} / \mathrm{kg}$ either as free (water-dissolved) fungicide or as clay (HT-OLE or HT-ELA)-based between 2 and 14 days, whereas those of $S$-metalaxyl ranged between 27 and 104 days (Table 
1 accordingly, $E F$ values, $E F=[S] /([S]+[R])$, rapidly became $>0.5$ after each application of the

2 fungicide (Figure 3). These results are consistent with previous studies indicating that the degradation of metalaxyl in soil is an enantioselective, microbially-mediated process, and that in aerobic soils with $\mathrm{pH}>5$, the fungicidally-active $R$-enantiomer of metalaxyl appears to be degraded faster than the $S$-enantiomer. ${ }^{12,13}$ Under a single $r a c$-metalaxyl application at a rate of $\sim 2.2 . \mathrm{mg} / \mathrm{kg}$ to surface soils with $\mathrm{pH}>5$, Buerge et al. ${ }^{12}$ reported half-lives in the range 422 days for $R$-metalaxyl and 23-50 days for $S$-metalaxyl. Our data further indicate that the preferential degradation of $R$-metalaxyl in soil remains upon repeated applications of the fungicide and is independent of whether the fungicide is applied as free (water-dissolved) or as clay-supported fungicide (Table 1).

Although neither the repeated application of the fungicide nor the type of formulation altered the " $R$-over- $S$ " preferential degradation of metalaxyl in the soil, both experimental variables affected the dissipation rate of metalaxyl enantiomers, thereby impacting the enantioselectivity of the metalaxyl degradation process (Table 1). To quantitatively describe the enantioselectivity of metalaxyl degradation under the different application regimes assayed in this study, we used the expression proposed by Müller and Buser, ${ }^{41}$ which takes into account the excess of the rate of the faster over the slower degraded enantiomers in a particular medium:

where $k_{1}$ and $k_{2}$ are the rate constants of the faster and slower degraded enantiomer, respectively. ${ }^{39,41,42}$ The $E S$ value can range between 0 (for a non-enantioselective process: $k_{2}=$ $k_{1}$ ) and 1 (for a fully enantioselective process: $k_{2}=0$ or $k_{2}<<k_{1}$ ). 
The $E S$ values for the degradation of metalaxyl under the different application conditions assayed in this study are included in Table 1. For all formulations, the ES value increased with the number of fungicide applications, indicating enhanced enantioselectivity of the metalaxyl degradation process. This was due to the fact that the repeated application of rac-metalaxyl to the soil led to a progressive reduction in the persistence of the biologically-active $R$-metalaxyl enantiomer (ANCOVA: $P<0.05$ ) and, simultaneously, to a progressive increase in the persistence of the non-active $S$-metalaxyl enantiomer (ANCOVA: $P<0.001$ for third $v s$ first application), so that the behavior of the two enantiomers became more and more divergent. For example, the half-life of $R$-metalaxyl in the soil decreased from 14 to 6 days after three successive applications of rac-metalaxyl as free (water dissolved) compound, while the halflife of $S$-metalaxyl increased from 34 to 104 days (Table 1). A similar effect was observed when the fungicide was applied as HT-OLE and HT-ELA formulations (Table 1). As a consequence, very high $E F$ values were reached shortly after the second and third fungicide applications as compared to the values obtained for the first application (Figure 3).

Accelerated degradation under repeated pesticide applications has been attributed to microbial adaptation leading to rapid metabolism, whereas slower degradation following repeated applications has been attributed to a number of mechanisms including toxicity of the pesticide or pesticide metabolites to soil biomass or lack of easily degradable organic matter in soil. ${ }^{22,43}$ A possible alternative explanation for the slower degradation of $S$-metalaxyl upon repeated applications (Figure 2, Table 1) is that, as a result of enhanced sorption with time, the amount of this enantiomer remaining after the first 42 days of incubation would degrade at a slower rate than the amount freshly applied to the soil during the second (and third) application, making the degradation biphasic. ${ }^{13}$ If so, the slow rate from the first application would contribute to slower first-order kinetic rate of the second application, and the slow 
1 biphasic rate from the first and second applications would then contribute to slower first-order

2 kinetic rate of the third application.

With regard to the type of formulation, the $R$ enantiomer was degraded faster when applied as HT-OLE and HT-ELA formulations as compared to the free (water-dissolved) unsupported enantiomer (ANCOVA: $P<0.05$ ) (Table 1). This was observed for all three successive applications conducted on the soil (Table 1). For the $S$ enantiomer, the differences between the dissipation rate constants were significant only for the third application (ANCOVA: $P<0.01$ ), where $S$-metalaxyl also degraded faster when applied as HT-OLE and HT-ELA formulations. The type of formulation (free $v s$ supported) had less impact on the ES value than the number of applications, because it affected the persistence of the two enantiomers in the same direction (Table 1).

The fact that the addition of metalaxyl pre-adsorbed on the organohydrotalcites not only did not retard the degradation of the fungicide in the soil, as expected from the slow release of the fungicide from the supports (Figure 1), but even accelerated the degradation process, indicated a very high bioavailability of HT-OLE- and HT-ELA-sorbed metalaxyl residues. Although sorbed organic compounds are generally considered as not directly available for microorganisms, ${ }^{44,45}$ it has also been suggested that certain sorbed substrates can be directly degraded by extracellular enzymes or biomass even when the enzyme/biomass itself is sorbed. ${ }^{46,47}$ In addition, the supports (HT-OLE and HT-ELA) could have positively affected the number and/or the activity of metalaxyl-degrading microorganisms or enzymes, for instance, by stimulating microbial growth, ${ }^{48}$ by adsorbing the degrader microorganisms, ${ }^{49}$ or by avoiding too high concentrations of the fungicide in the aqueous phase that could exert an adverse (toxic) effect on the soil microbial population. ${ }^{29,50}$ The interactions of organoclays with microbial growth and activity in soil are poorly documented, so that additional 
1 information is needed on this subject to better understand the soil functioning in the presence

2 of this kind of exogenous material. ${ }^{48,51}$

3

4

5

\subsection{Metalaxyl leaching}

Figures 4 and 5 show the breakthrough curves (BTCs) for $S$ - and $R$-metalaxyl obtained in the column leaching experiment. During the experiment, rac-metalaxyl was successively applied to soil columns three times at a rate of $2 \mathrm{~kg} / \mathrm{ha}$, either as free (water-dissolved) fungicide or as the clay (HT-OLE/HT-ELA)-based formulations. The maximum concentrations of $S$ - and $R$ metalaxyl in leachates, total amounts of $S$ - and $R$-metalaxyl leached, and average $E F$ values in leachates at two representative regions of the BTCs are summarized in Table 2.

For all applications assayed, the maximum concentrations of $S$ - and $R$-metalaxyl appeared in leachates after applying a similar amount of water, which ranged between 90 and $120 \mathrm{~mL}$ or 1.6-2.1 times the pore volume $\left(\mathrm{V}_{\mathrm{p}}\right)$ of the soil column (Figure 4). The similar retardation of $S$ - and $R$-metalaxyl reflected that sorption by the soil was non-enantioselective, because significant enantioselective sorption would have resulted in one enantiomer being leached earlier than the other. ${ }^{13}$ Nevertheless, the concentration of $S$-metalaxyl in all leachates collected was greater than that of $R$-metalaxyl (Figure 4), resulting in leachates with $E F$ values $>0.5$ (Table 2). As a consequence, total cumulative amounts of $S$-enantiomer leached were greater than those of $R$-enantiomer (Figure 5, Table 2). The preferential degradation of $R$-metalaxyl within the soil column reduced the amount of this enantiomer in leachates, yielding an apparent enantioselective leaching of the fungicide.

As observed in the incubation experiment, both the repeated application of the fungicide and the type of formulation impacted the leaching profiles of $S$ - and $R$-metalaxyl. Application of the fungicide as organohydrotalcite-based formulations resulted in flattening of the BTCs of both enantiomers and shifting of the maximum concentration peaks of the BTCs to larger 
1 water volumes as compared to the application of the free (water-dissolved) fungicide (Figure

2 4). This behavior can be attributed to the sustained release of metalaxyl enantiomers from the clay-based formulations (Figure 1b). The total amounts of $S$ - and $R$-metalaxyl leached for the clay-based formulations were less than those for the unsupported fungicide (Figure 5, Table 2). This could have been due to: i) a longer residence time of the enantiomers within the soil column as a result of their slow release from the supports (Figure 1b) and ii) the fast degradation of the two enantiomers when applied to soil as clay-based formulations (Figure 2, Table 1).

The most evident effect of the number of applications on the BTCs of $S$ - and $R$-metalaxyl was a progressive reduction in the leachate concentrations of $R$-metalaxyl as the number of applications was increased. In contrast, the leachate concentrations of $S$-metalaxyl were mostly unaffected, so that the EF values of the leachates increased with the number of fungicide applications (Figure 4, Table 2). Again, this behavior reflected the degradation patterns of metalaxyl enantiomers in soil under repeated fungicide applications. The enhanced degradation of $R$-metalaxyl in soil pre-treated with the fungicide reduced the concentrations of this enantiomer in leachates and in turn the total amounts leached (Figures 4 and 5). The effect of repeated applications on the leaching of $S$-metalaxyl was less evident most likely because the residence time of this enantiomer within the soil column (generally less than 15 days) was short compared to its soil half-life (Table 1), and hence, was not enough to develop differences in degradation between the first and subsequent applications of the fungicide to the soil columns.

\section{CONCLUSIONS}

Repeated applications and the type of formulation affected the enantioselectivity of the dissipation and leaching processes of metalaxyl in a slightly alkaline, loamy sand soil. 
1 Repeated applications of metalaxyl progressively reduced the persistence of $R$-metalaxyl and,

2 simultaneously, increased the persistence of $S$-metalaxyl, while the enantiomers generally degraded faster when applied as clay-based formulations than when applied as free (waterdissolved) compounds. The different release and degradation patterns of $S$ - and $R$-metalaxyl under the different application regimes assayed affected the leaching behavior of the fungicide. The clay-based formulations reduced the leaching of both enantiomers and repeated fungicide applications led to leachates progressively enriched in the $S$-enantiomer. On the basis of the observed effects, the repeated application of metalaxyl to the same soil may have the adverse effect of shortening the biological efficacy of the fungicidally-active $R$ enantiomer with a simultaneous accumulation of residues corresponding to the non-active $S$ enantiomer. Application of the fungicide as clay-based formulations can be a good practice to reduce the persistence and leaching of the two metalaxyl enantiomers, bearing in mind that the fast degradation of the $R$ enantiomer when supported on clays might also shorten the biological efficacy of the formulation, particularly upon repeated applications. Although degradation products were not monitored in the present study, previous work has indicated that the microbial degradation of metalaxyl enantiomers in soil may lead to accumulation of the major metabolite "metalaxyl-acid", whose formation occurs with retention of configuration. ${ }^{12,39}$ Therefore, addressing the fate of $(R)$-metalaxyl acid in soils similar to that used in the present study, subjected to repeated applications of metalaxyl, may become particularly relevant, since, due to its acidic character, metalaxyl-acid is expected to display lower sorption and higher mobility than the parent compound in heavy-textured, neutral and alkaline soils. $^{52}$ 


\section{ACKNOWLEDGEMENTS}

2 This work has been financed by the Spanish Ministry of Economy and Competitiveness

3 (MINECO Project AGL2011-23779) and Junta de Andalucía (Research Group AGR-264),

4 cofinanced with European FEDER-FSE funds (Operative Program 2007-2013). B. Gámiz

5 thanks JA for a post-doctoral contract linked to the Project P07-AGR-03077. M.A. Adelino

6 thanks MINECO for a pre-doctoral FPI fellowship. The authors also thank M.J. Calderón

7 (IRNAS, CSIC), M.I. Cardo (IRNAS, CSIC), and P. Franco (Chiral Technologies Europe) for

8 technical assistance and Dr. S. Redondo and Dr. E. Mateos (Univ. of Seville) for their help

9 with the statistical treatment of the data. 


\section{REFERENCES}

1 Magrans JO, Alonso-Prados JL and García-Baudín JM, Importance of considering pesticide stereoisomerism-proposal of a scheme to apply Directive 91/414/CEE framework to pesticide active substances manufactured as isomeric mixtures. Chemosphere 49:461-469 (2002).

2 Garrison AW, Probing the enantioselectivity of chiral pesticides. Environmental Science \& Technology 40:16-23 (2006).

3 Pérez S and Barceló D, Applications of LC-MS to quantitation and evaluation of the environmental fate of chiral drugs and their metabolites. Trac-Trends in Analytical Chemistry 27:836-846 (2008).

4 Ye J, Zhao M, Liu J and Liu W, Enantioselectivity in environmental risk assessment of modern chiral pesticides. Environmental Pollution 158:2371-2383 (2010).

5 Williams A, Opportunities for chiral agrochemicals. Pesticide Science 46:3-9 (1996).

6 Chen S and Liu W, Toxicity of chiral pesticide rac-metalaxyl and $R$-metalaxyl to Daphnia magna. Bulletin of Environmental Contamination and Toxicology 81:531$534(2008)$.

7 Wong CS, Environmental fate processes and biochemical transformations of chiral emerging organic pollutants. Analytical and Bioanalytical Chemistry 386:544-558 (2006).

8 Ramezani MK, Oliver DP, Kookana RS, Lao W, Gill G and Presto C, Faster degradation of herbicidally-active enantiomer of imidazolinones in soils. Chemosphere 79:1040-1045 (2010).

9 Buser HR, Müller MD and Rappe C, Enantioselective determination of chlordane components using chiral high-resolution gas chromatography-mass spectrometry 
with application to environmental samples. Environmental Science \& Technology 26:1533-1540 (1992).

10 Garrison AW, Schmitt P, Martens D and Kettrup A, Enantiomeric selectivity in the environmental degradation of dichlorprop as determined by high-performance capillary electrophoresis. Environmental Science \& Technology 30:2449-2455 (1996).

11 Romero E, Matallo MB, Peña A, Sánchez-Rasero F, Schmitt-Kopplin Ph and Dios G, Dissipation of racemic mecoprop and dichlorprop and their pure R-enantiomers in three calcareous soils with and without peat addition. Environmental Pollution 111:209-215 (2001).

12 Buerge IJ, Poiger T, Müller MD and Buser HR, Enantioselective degradation of metalaxyl in soils: chiral preference changes with soil pH. Environmental Science \& Technology 37:2668-2674 (2003).

13 Celis, R, Gámiz B, Adelino MA, Hermosín MC and Cornejo J, Environmental behavior of the enantiomers of the chiral fungicide metalaxyl in Mediterranean agricultural soils. Science of the Total Environment 444:288-297 (2013).

14 Wedyan M and Preston MR, Isomer-selective adsorption of amino acids by components of natural sediments. Environmental Science \& Technology 39:21152119 (2005).

15 Liu $\mathrm{Z}$ and Lee $\mathrm{C}$, The role of organic matter in the sorption capacity of marine sediments. Marine Chemistry 105:240-257 (2007).

16 Oravec M, Simek $\mathrm{Z}$ and Holoubek I, The effect of humic acid and ash on enantiomeric fraction change of chiral pollutants. Colloids and Surfaces A 359:60-65 (2010). 
17 Zipper C, Suter MJF, Haderlein SB, Gruhl M and Kohler HPE, Changes in the enantiomeric ratio of (R)- to (S)-mecoprop indicate in situ biodegradation of this chiral herbicide in a polluted aquifer. Environmental Science \& Technology 32:20702076 (1998).

18 Jarman JL, Jones WJ, Howell LA and Garrison AW, Application of capillary electrophoresis to study the enantioselective transformation of five chiral pesticides in aerobic soil slurries. Journal of Agricultural and Food Chemistry 53:6175-6182 (2005).

19 Chen S and Liu W, Enantioselective degradation of metalaxyl in anaerobic activated sewage sludge. Bulletin of Environmental Contamination and Toxicology 82:327-331 (2009).

20 Sukul P, Lamshoft M, Zuhlke S and Spiteller M, Evaluation of sorption-desorption processes for metalaxyl in natural and artificial soils. Journal of Environmental Science and Health Part B 48:431-441 (2013).

21 Walker A and Welch SJ, Enhanced degradation of some soil-applied herbicides. Weed Research 31:49-57 (1991).

22 Cox L, Walker A and Welch SJ. Evidence for the accelerated degradation of isoproturon in soils. Pesticide Science 48:253-260 (1996).

23 Cox L, Celis R, Hermosín MC and Cornejo J, Natural soil colloids to retard simazine and 2,4-D leaching in soil. Journal of Agricultural and Food Chemistry 48:93-99 (2000).

24 Ismail BS and Kalithasan K, Effect of repeated application on persistence and downward movement of four herbicides in soil. Australian Journal of Soil Research 35:503-513 (1997). 
25 Abdelhafid R, Houot S and Barriuso E, Dependence of atrazine degradation on C and $\mathrm{N}$ availability in adapted and non-adapted soils. Soil Biology and Biochemistry 32:389-401 (2000).

26 Hermosín MC, Calderón MJ, Aguer JP and Cornejo J, Organoclays for controlled release of the herbicide fenuron. Pest Management Science 57:803-809 (2001).

27 Papini S and de Andréa MM, Enhanced degradation of metalaxyl in agricultural soils of Sao Paulo State, Brazil. Pesquisa Agropecuaria Brasileira 36:1-5 (2001).

28 Sopeña F, Maqueda C and Morillo E, Norflurazon mobility, dissipation, activity, and persistence in a sandy soil as influenced by formulation. Journal of Agricultural and Food Chemistry 55:3561-3567 (2007).

29 Vischetti C, Monaci E, Cardinali A, Casucci C and Perucci P, The effect of initial concentration, co-application and repeated applications on pesticide degradation in a biobed mixture. Chemosphere 72:1739-1743 (2008).

30 Bruna F, Pavlovic I, Celis R, Barriga C, Cornejo J and Ulibarri MA, Organohydrotalcites as novel supports for the slow release of the herbicide terbuthylazine. Applied Clay Science 42, 194-200 (2008).

31 Monkiedje A and Spiteller M, Degradation of metalaxyl and mefenoxam and effects on the microbiological properties of tropical and temperate soils. International Journal of Environmental Research and Public Health 2:272-285 (2005).

32 Monkiedje A, Zuehlke S, Jacqueline S, Maniepi N and Spiteller M, Elimination of racemic and enantioenriched metalaxyl based fungicides under tropical conditions in the field. Chemosphere 69, 655-663 (2007).

33 Celis R, Adelino MA, Gámiz B, Hermosín MC, Koskinen WC and Cornejo J, Nanohybrids of $\mathrm{Mg} / \mathrm{Al}$ layered double hydroxide and long-chain (C18) unsaturated 
fatty acid anions: structure and sorptive properties. Applied Clay Science, 96, 81-90 (2014).

34 Celis R, Koskinen WC, Cecchi AM, Bresnahan GA, Carrisoza MJ, Ulibarri M, Pavlovic I and Hermosín MC, Sorption of the ionizable pesticide imazamox by organo-clays and organohydrotalcites. Journal of Environmental Science and Health Part B 34:929-941 (1999).

35 Cornejo J, Celis R, Pavlovic I and Ulibarri MA, Interactions of pesticides with clays and layered double hydroxides: a review. Clay Minerals 43:155-175 (2008).

36 Chuang YH, Liu CH, Tzou YM, Chang JS, Chiang PN and Wang MK, Comparison and characterization of chemical surfactants and biosurfactants intercalated with layered double hydroxides (LDHs) for removing naphthalene from contaminated aqueous solutions. Colloids and Surfaces A 366:170-177 (2010).

37 Bruna F, Celis R, Real M and Cornejo J, Organo/LDH nanocomposite as an adsorbent of polycyclic aromatic hydrocarbons in water and soil-water systems. Journal of Hazardous Materials 225-226:74-80 (2012).

38 Cavani F, Tirifò F and Vaccari A, Hydrotalcite-type anionic clays: preparation, properties and applications. Catalysis Today 11, 173-301 (1991).

39 Buser HR, Müller MD, Poiger T and Balmer ME, Environmental behavior of the chiral acetamide pesticide metalaxyl: enantioselective degradation and chiral stability in soil. Environmental Science \& Technology 36:221-226 (2002).

40 Harner T, Wiberg K and Norstrom R, Enantiomer fractions are preferred to enantiomer ratios for describing chiral signatures in environmental analysis. Environmental Science \& Technology 34:218-220 (2000). 
41 Müller MD and Buser HR, Environmental behavior of acetamide pesticide stereoisomers. 2. Stereo- and enantioselective degradation in sewage sludge and soil. Environmental Science \& Technology 29:2031-2037 (1995).

42 Gámiz B, Celis R, Hermosín MC and Cornejo J. Effect of olive-mill waste addition to agricultural soil on the enantioselective behavior of the chiral fungicide metalaxyl. Journal of Environmental Management 128:92-99 (2013).

43 Motonaga K, Takagi K, Matumoto S, Suppression of chlorothalonil degradation in soil after repeated application. Environmental Toxicology and Chemistry 17:14691472 (1998).

44 Ogram AV, Jessup RE, Ou LT and Rao PSC, Effects of sorption on biological degradation rates of (2,4-dichloro-phenoxy)-acetic acid in soils. Applied Environmental Microbiology 49:582-587 (1985).

45 Shelton DR and Doherty MA, A model describing pesticide bioavailability and biodegradation in soil. Soil Science Society of America Journal 61:1078-1084 (1997).

46 Park JH, Kay D, Zhao XD, Boyd SA and Voice TC, Kinetic modeling of bioavailability of sorbed-phase 2,4-dichlorophenoxyacetic acid. Journal of Environmental Quality 30:1523-1527 (2001).

47 Singh N, Megharaj M, Gates WP, Churchman GJ, Anderson J, Kookana RS, Naidu R, Chen Z, Slade PG and Sethunathan N, Bioavailability of an organophosphorus pesticide, fenamiphos, sorbed on an organo-clay. Journal of Agricultural and Food Chemistry 51:2653-2658 (2003).

48 Abbate C, Ambrosoli R, Minati JL, Gennari M and Arena M., Metabolic and molecular methods to evaluate the organoclay effects on a bacterial community. Environmental Pollution 179:39-44 (2013). 
49 Alekseevaa T, Prevotb V, Sancelme M, Forano C, Besse-Hoggan P, Enhancing atrazine biodegradation by Pseudomonas sp. strain ADP adsorption to Layered Double Hydroxide bionanocomposites. Journal of Hazardous Materials 191:126-135 (2011).

50 Helweg A, Fomsgaard IS, Reffstrup TK and Sorensen H, Degradation of mecoprop and isoproturon in soil: influence of initial concentration. International Journal of Environmental Analytical Chemistry 70:133-148 (1998).

51 Abbate C, Arena M, Baglieri A and Gennari M, Effects of organoclays on soil eubacterial community assessed by molecular approaches. Journal of Hazardous Materials 168:466-472 (2009).

52 Monkiedje A, Spiteller M, Sorptive behavior of the phenylamide fungicides, mefenoxam and metalaxyl, and their acid metabolite in typical Cameroonian and German soils. Chemosphere 49:659-668 (2002). 


\section{FIGURE CAPTIONS}

2 Figure 1. Sorption isotherms of rac-metalaxyl on hydrotalcite (HT), oleate-modified hydrotalcite (HT-OLE), and elaidate-modified hydrotalcite (HT-ELA) (a), and metalaxyl release kinetics into water from its complexes with HT-OLE and HT-ELA (b). Data in the graphs correspond to the sum of the $R+S$ metalaxyl sorbed and released. Error bars correspond to the standard error of triplicate measurements.

Figure 2. $S$ - and $R$-metalaxyl dissipation curves upon three successive applications of racmetalaxyl to soil as free compound and as HT-OLE and HT-ELA formulations. Symbols represent experimental data points, solid lines correspond to the fittings to first-order dissipation kinetics, and $\mathrm{t}_{0}, \mathrm{t}_{0}{ }^{\prime}$ and $\mathrm{t}_{0}{ }^{\prime \prime}$ indicate, respectively, the times at which the first, second and third applications of metalaxyl were carried out.

Figure 3. Changes in the enantiomer fraction (EF) with time during the incubation experiment. Error bars correspond to the standard error of triplicate measurements and $t_{0}$, $\mathrm{t}_{0}{ }^{\prime}$ and $\mathrm{t}_{0}{ }^{\prime \prime}$ indicate, respectively, the times at which the first, second, and third applications of metalaxyl were carried out.

Figure 4. Breakthrough curves for $S$ - and $R$-metalaxyl in soil columns upon three successive applications of rac-metalaxyl as free compound and as HT-OLE and HT-ELA formulations. Error bars correspond to standard errors of triplicate measurements and $\mathrm{t}_{0}$, $\mathrm{t}_{0}$ 'and $\mathrm{t}_{0}{ }^{\prime \prime}$ indicate, respectively, the times at which the first, second, and third applications of metalaxyl were carried out. 
1 Figure 5. Cumulative breakthrough curves for $S$ - and $R$-metalaxyl in soil columns upon three

2 successive applications of rac-metalaxyl as free compound and as HT-OLE and HT-ELA

3 formulations. Error bars correspond to standard errors of triplicate measurements and $\mathrm{t}_{0}$,

$4 \mathrm{t}_{0}{ }^{\prime}$ and $\mathrm{t}_{0}{ }^{\prime \prime}$ indicate, respectively, the times at which the first, second, and third applications of

5 metalaxyl were carried out. 
Table 1. First-order dissipation constants $\left(\mathrm{C}_{0}\right.$ and $\left.k\right)$, determination coefficients for the linear fits $\left(R^{2}\right)$, and half-lives $\left(\mathrm{t}_{1 / 2}\right)$ for $S$ - and $R$ metalaxyl in soil under three successive applications of rac-metalaxyl as free compound and as HT-OLE and HT-ELA formulations.

\begin{tabular}{|c|c|c|c|c|c|c|c|c|c|}
\hline \multirow[t]{2}{*}{ Application } & \multicolumn{4}{|c|}{$S$-metalaxyl } & \multicolumn{4}{|c|}{$R$-metalaxyl } & \multirow[b]{2}{*}{$E S^{b}$} \\
\hline & $\begin{array}{c}\mathrm{C}_{0} \\
(\mathrm{mg} / \mathrm{kg})\end{array}$ & $\begin{array}{c}k \\
\left(\text { days }^{-1}\right)\end{array}$ & $R^{2}$ & $\begin{array}{c}\mathrm{t}_{1 / 2} \\
\text { (days) }\end{array}$ & $\begin{array}{c}\mathrm{C}_{0} \\
(\mathrm{mg} / \mathrm{kg})\end{array}$ & $\begin{array}{c}k \\
\left(\text { days }^{-1}\right)\end{array}$ & $R^{2}$ & $\begin{array}{c}\mathrm{t}_{1 / 2} \\
\text { (days) }\end{array}$ & \\
\hline \multicolumn{10}{|c|}{ Free } \\
\hline $1 \mathrm{st}$ & $0.98(0.95-1.00)^{a}$ & $0.020 \pm 0.001^{a}$ & 0.99 & $34 \pm 2^{a}$ & $1.02(0.98-1.05)$ & $0.048 \pm 0.002$ & 0.99 & $14 \pm 1$ & 0.41 \\
\hline 2nd & $1.17(1.15-1.20)$ & $0.014 \pm 0.001$ & 0.97 & $50 \pm 5$ & $1.01(0.96-1.06)$ & $0.071 \pm 0.003$ & 0.99 & $10 \pm 1$ & 0.67 \\
\hline $3 \mathrm{rd}$ & $1.91(1.88-1.94)$ & $0.007 \pm 0.001$ & 0.90 & $104 \pm 12$ & $1.42(1.31-1.53)$ & $0.108 \pm 0.005$ & 0.98 & $6 \pm 1$ & 0.88 \\
\hline \multicolumn{10}{|c|}{ HT-OLE } \\
\hline $1 \mathrm{st}$ & $1.04(0.98-1.11)$ & $0.025 \pm 0.003$ & 0.94 & $27 \pm 3$ & $1.32(1.08-1.61)$ & $0.080 \pm 0.010$ & 0.92 & $9 \pm 1$ & 0.52 \\
\hline 2nd & $1.09(1.03-1.17)$ & $0.016 \pm 0.004$ & 0.84 & $45 \pm 15$ & $0.99(0.88-1.10)$ & $0.240 \pm 0.015$ & 0.99 & $3 \pm 1$ & 0.88 \\
\hline $3 r d$ & $1.73(1.69-1.76)$ & $0.010 \pm 0.001$ & 0.95 & $68 \pm 6$ & $1.10(1.08-1.12)$ & $0.381 \pm 0.006$ & 1.00 & $2 \pm 1$ & 0.95 \\
\hline \multicolumn{10}{|c|}{ HT-ELA } \\
\hline $1 \mathrm{st}$ & $0.90(0.87-0.93)$ & $0.024 \pm 0.002$ & 0.97 & $29 \pm 2$ & $1.02(0.98-1.06)$ & $0.055 \pm 0.002$ & 0.99 & $13 \pm 1$ & 0.39 \\
\hline 2nd & $1.14(1.08-1.19)$ & $0.019 \pm 0.003$ & 0.90 & $36 \pm 8$ & $1.09(0.92-1.28)$ & $0.121 \pm 0.011$ & 0.97 & $6 \pm 1$ & 0.73 \\
\hline $3 \mathrm{rd}$ & $1.79(1.75-1.83)$ & $0.013 \pm 0.001$ & 0.94 & $53 \pm 4$ & $1.12(1.02-1.23)$ & $0.433 \pm 0.023$ & 0.99 & $2 \pm 1$ & 0.94 \\
\hline
\end{tabular}

${ }^{a}$ standard error interval.

${ }^{b} E S=\left(k_{\mathrm{R}}-k_{\mathrm{S}}\right) /\left(k_{\mathrm{R}}+k_{\mathrm{S}}\right)$ 
Table 2. Summary of $S$ - and $R$-metalaxyl column leaching data extracted from the breakthrough curves (BTCs) of rac-metalaxyl applied to the soil columns as free compound and as HT-OLE and HT-ELA formulations.

\begin{tabular}{|c|c|c|c|c|c|c|}
\hline \multirow{4}{*}{ Application } & \multicolumn{2}{|c|}{$S$-metalaxyl } & \multicolumn{2}{|c|}{$R$-metalaxyl } & \multirow{4}{*}{$\mathrm{EF}_{1}^{b}$} & \multirow{4}{*}{$\mathrm{EF}_{2}{ }^{c}$} \\
\hline & $\mathrm{C}_{\max }{ }^{a}$ & Total & $\mathrm{C}_{\max }{ }^{a}$ & Total & & \\
\hline & & leached & & \multirow{2}{*}{$\begin{array}{c}\text { leached } \\
\qquad \%)\end{array}$} & & \\
\hline & $(\mathrm{mg} / \mathrm{L})$ & $(\%)$ & $(\mathrm{mg} / \mathrm{L})$ & & & \\
\hline \multicolumn{7}{|c|}{ Free } \\
\hline 1 st & $1.27 \pm 0.05^{d}$ & $92 \pm 1$ & $1.17 \pm 0.09$ & $82 \pm 1$ & $0.52 \pm 0.01$ & $0.56 \pm 0.01$ \\
\hline $2 n d$ & $1.40 \pm 0.21$ & $91 \pm 1$ & $1.26 \pm 0.08$ & $78 \pm 1$ & $0.53 \pm 0.01$ & $0.61 \pm 0.01$ \\
\hline $3 \mathrm{rd}$ & $1.39 \pm 0.10$ & $92 \pm 1$ & $1.14 \pm 0.09$ & $71 \pm 2$ & $0.55 \pm 0.01$ & $0.64 \pm 0.02$ \\
\hline \multicolumn{7}{|c|}{ HT-OLE } \\
\hline 1 st & $0.55 \pm 0.02$ & $70 \pm 2$ & $0.50 \pm 0.01$ & $62 \pm 1$ & $0.53 \pm 0.01$ & $0.56 \pm 0.01$ \\
\hline $2 n d$ & $0.56 \pm 0.03$ & $86 \pm 5$ & $0.46 \pm 0.03$ & $67 \pm 4$ & $0.55 \pm 0.01$ & $0.59 \pm 0.01$ \\
\hline $3 r d$ & $0.59 \pm 0.08$ & $83 \pm 8$ & $0.44 \pm 0.06$ & $58 \pm 5$ & $0.58 \pm 0.01$ & $0.62 \pm 0.01$ \\
\hline \multicolumn{7}{|c|}{ HT-ELA } \\
\hline $1 \mathrm{st}$ & $0.65 \pm 0.03$ & $75 \pm 2$ & $0.60 \pm 0.03$ & $67 \pm 2$ & $0.52 \pm 0.01$ & $0.56 \pm 0.01$ \\
\hline 2nd & $0.63 \pm 0.02$ & $82 \pm 3$ & $0.53 \pm 0.05$ & $65 \pm 3$ & $0.55 \pm 0.01$ & $0.60 \pm 0.01$ \\
\hline $3 r d$ & $0.60 \pm 0.04$ & $82 \pm 6$ & $0.45 \pm 0.04$ & $56 \pm 4$ & $0.58 \pm 0.01$ & $0.63 \pm 0.01$ \\
\hline \multicolumn{7}{|c|}{${ }^{a} \mathrm{C}_{\max }:$ maximum concentration found in leachates. } \\
\hline \multicolumn{7}{|c|}{$\begin{array}{l}{ }^{b} \mathrm{EF}_{1} \text { : average enantiomer fraction in leachates containing the three highest concentrations of } S \text { - and } R \text { - } \\
\text { metalaxyl. }\end{array}$} \\
\hline
\end{tabular}



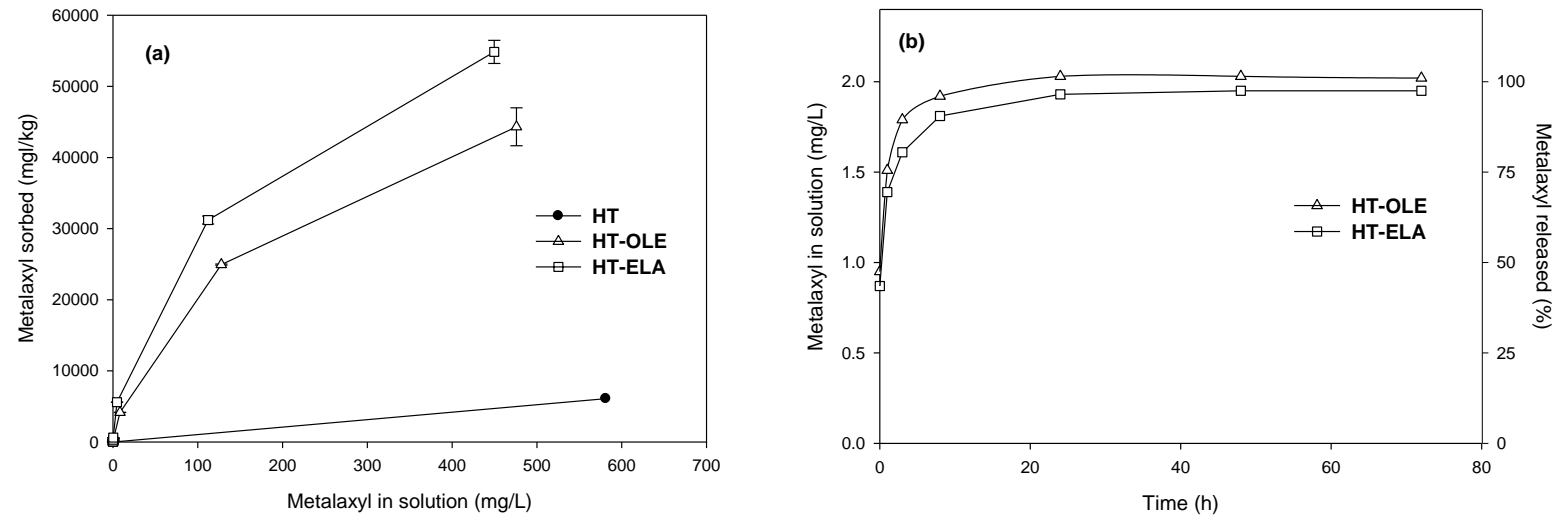

Figure 1. Sorption isotherms of rac-metalaxyl on hydrotalcite (HT), oleate-modified hydrotalcite (HT-OLE), and elaidate-modified hydrotalcite (HT-ELA) (a), and metalaxyl release kinetics into water from its complexes with HT-OLE and HT-ELA (b). Data in the graphs correspond to the sum of the $R+S$ metalaxyl sorbed and released. Error bars correspond to the standard error of triplicate measurements. 

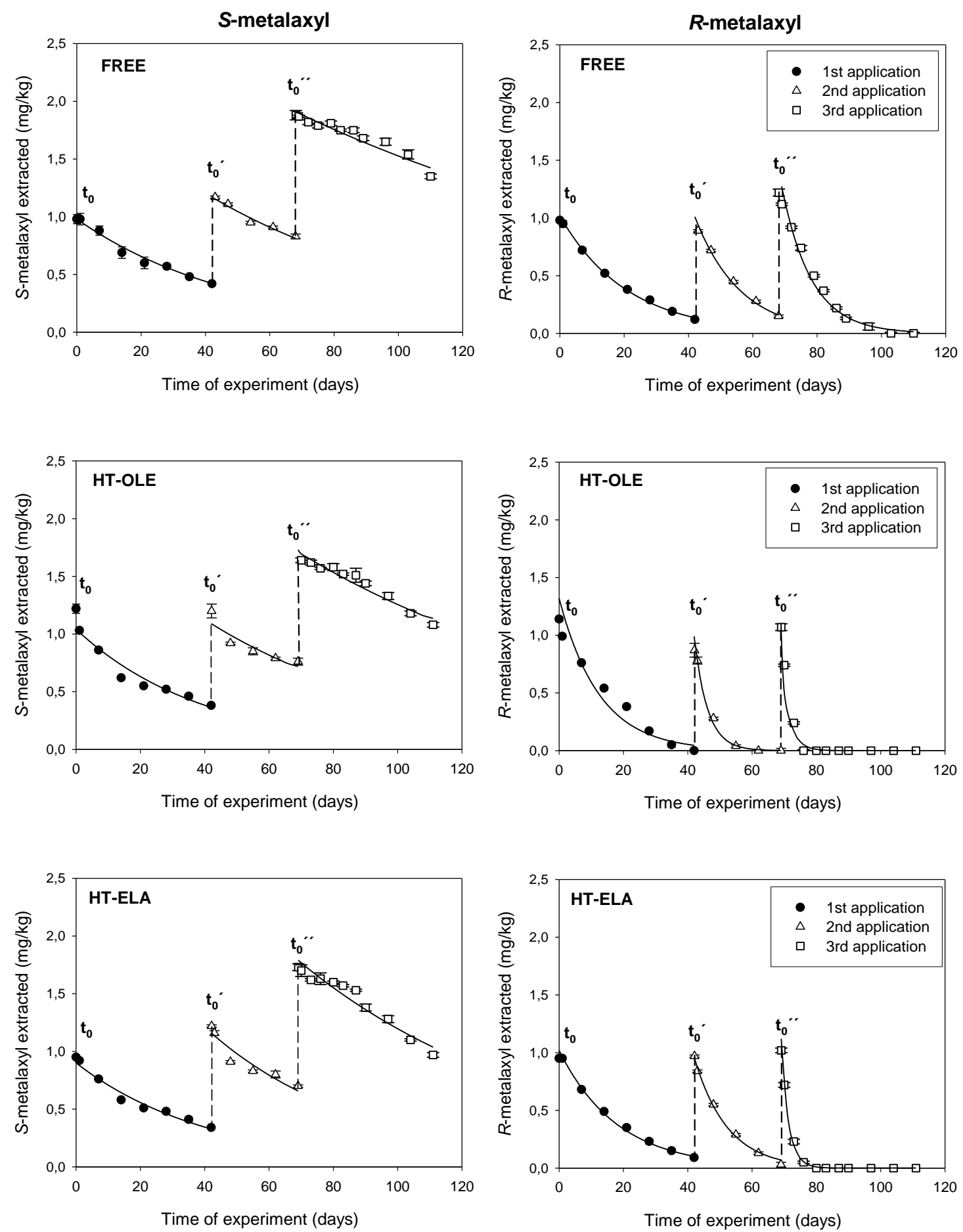

Figure 2. $S$ - and $R$-metalaxyl dissipation curves upon three successive applications of racmetalaxyl to soil as free compound and as HT-OLE and HT-ELA formulations. Symbols represent experimental data points, solid lines correspond to the fittings to first-order dissipation kinetics, and $\mathrm{t}_{0}, \mathrm{t}_{0}{ }^{\prime}$ and $\mathrm{t}_{0}{ }^{\prime \prime}$ indicate, respectively, the times at which the first, second and third applications of metalaxyl were carried out. 

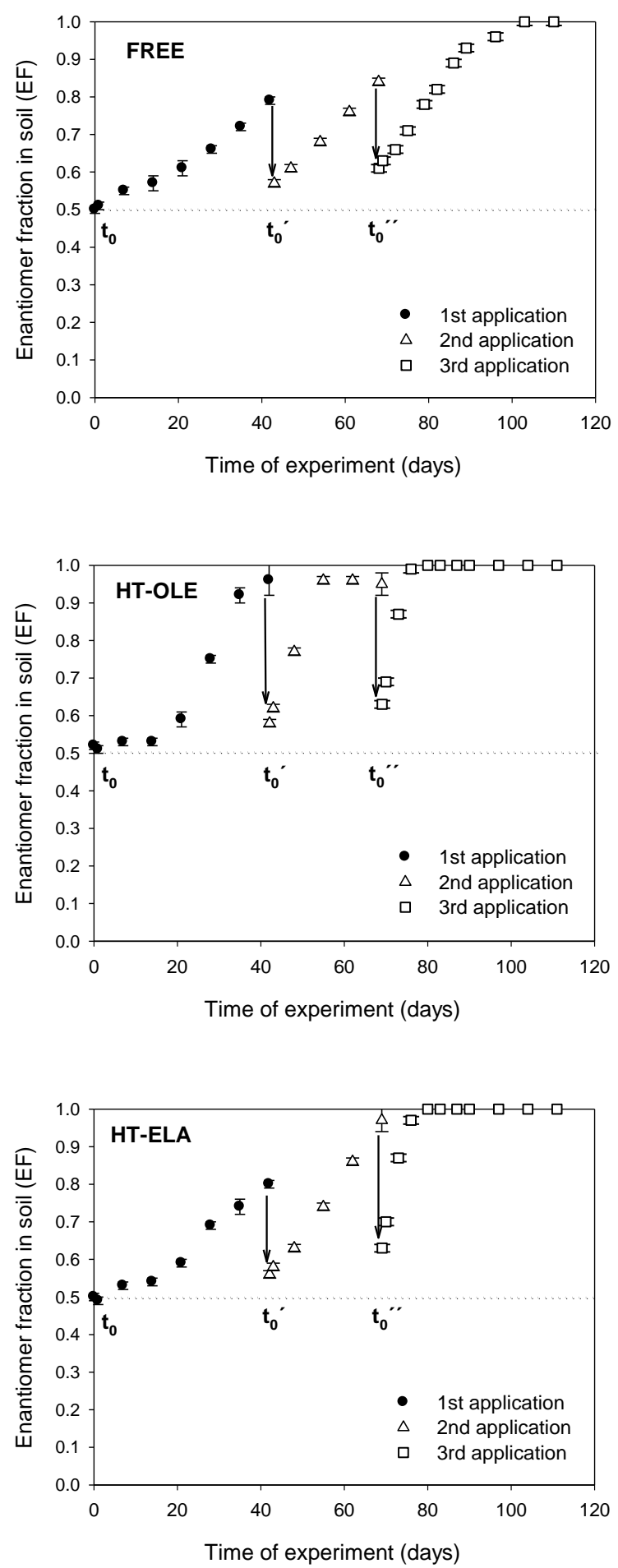

Figure 3. Changes in the enantiomer fraction (EF) with time during the incubation experiment. Error bars correspond to the standard error of triplicate measurements and $t_{0}$, $\mathrm{t}_{0}{ }^{\prime}$ and $\mathrm{t}_{0}{ }^{\prime \prime}$ indicate, respectively, the times at which the first, second, and third applications of metalaxyl were carried out. 

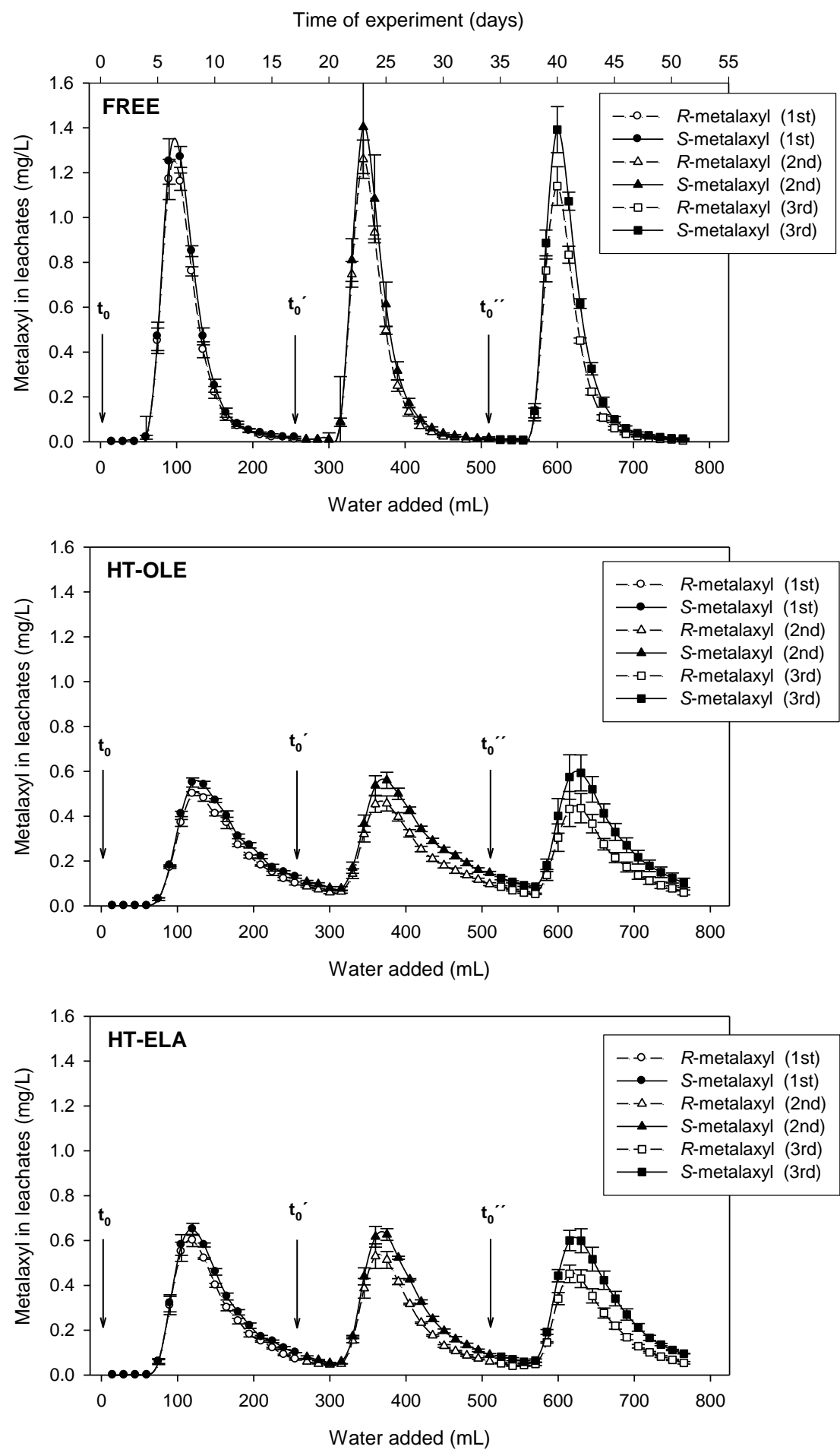

Figure 4. Breakthrough curves for $S$ - and $R$-metalaxyl in soil columns upon three successive applications of rac-metalaxyl as free compound and as HT-OLE and HT-ELA formulations. Error bars correspond to standard errors of triplicate measurements and $\mathrm{t}_{0}$, $\mathrm{t}_{0}$ ' and $\mathrm{t}_{0}{ }^{\prime \prime}$ indicate, respectively, the times at which the first, second, and third applications of metalaxyl were carried out. 

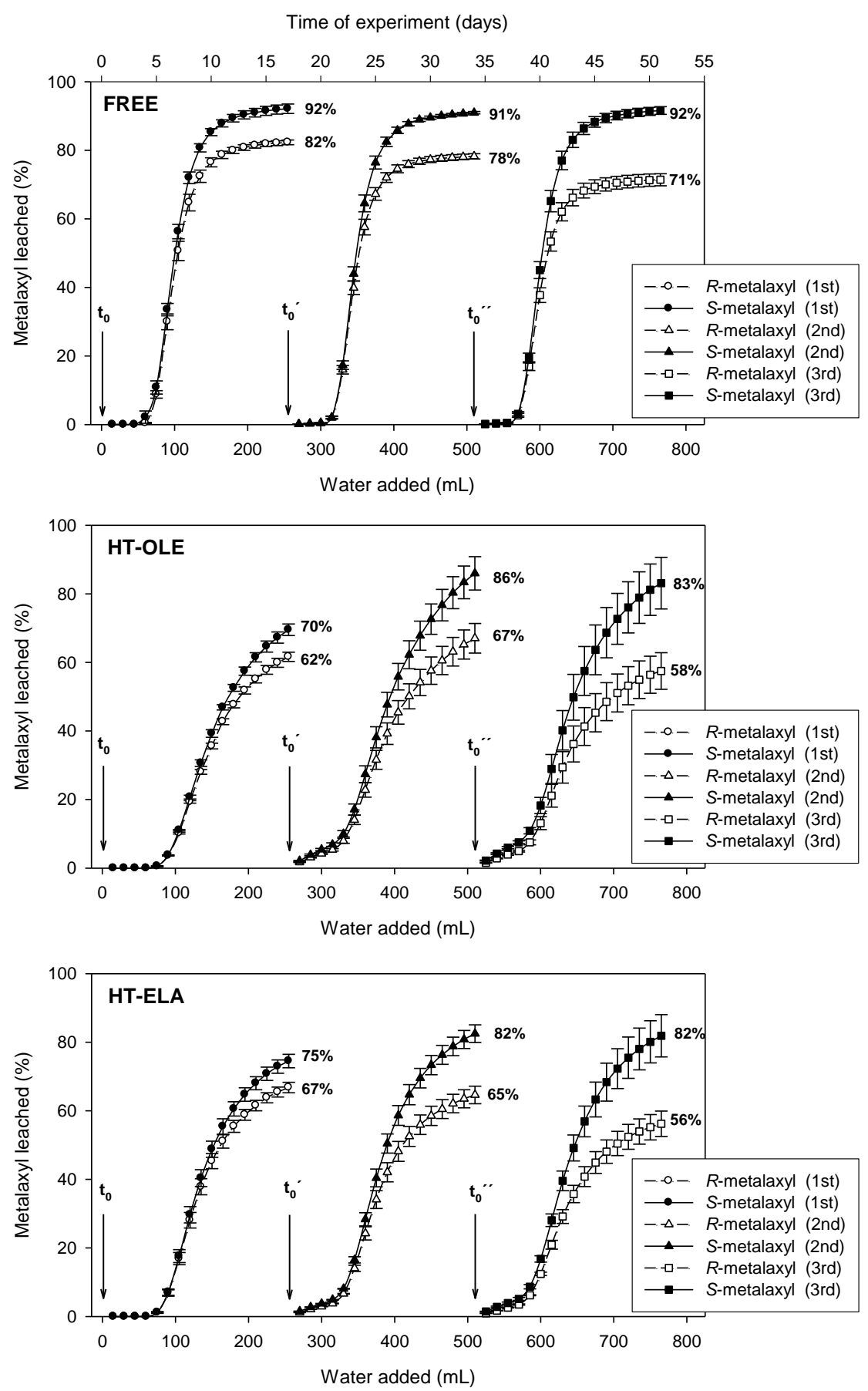

Figure 5. Cumulative breakthrough curves for $S$ - and $R$-metalaxyl in soil columns upon three successive applications of rac-metalaxyl as free compound and as HT-OLE and HTELA formulations. Error bars correspond to standard errors of triplicate measurements and $\mathrm{t}_{0}, \mathrm{t}_{0}$ 'and $\mathrm{t}_{0}{ }^{\prime \prime}$ indicate, respectively, the times at which the first, second, and third applications of metalaxyl were carried out. 